\title{
Moverse con dificultad en el gimnasio: consideraciones en torno a un problema educativo y de salud
}

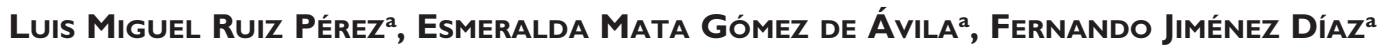 \\ y Juan Antonio Moreno Murciab,
}

aUniversidad de Castilla-La Mancha. Toledo. España.

bUniversidad de Murcia. Murcia. España.

\begin{abstract}
RESUMEN
Los problemas evolutivos de coordinación motriz en las edades escolares siguen siendo una dificultad oculta en el contexto español. Su impacto en las dimensiones física, social y emocional de los escolares ha sido destacado por los investigadores, y la necesidad de tomar conciencia de su presencia y de su efecto se hace muy necesaria. En este artículo se analiza en qué consiste esta dificultad, sus posibles causas y sus efectos, para destacar la necesidad de una intervención temprana para remediar sus consecuencias, $y$ en especial en la condición física de los escolares que la padecen, dada su tendencia hacia el sedentarismo y un estilo de vida poco activo. En definitiva, se destaca que los problemas evolutivos de coordinación motriz no son estrictamente un problema educativo, sino que se han convertido en un problema de salud que implica a todos en su solución.
\end{abstract}

PALABRAS CLAVE: Competencia motriz. Problemas evolutivos de coordinación motriz. Escolares. Actividad física y deporte.

\begin{abstract}
Developmental coordination disorder in schoolchildren is a hidden problem in Spain. The impact of this condition on the emotional, social and physical dimensions of children's development has been demonstrated and awareness of its effects and prevalence should be increased.

In this article we analyze the characteristics of this disorder, its possible causes, and its effects, with the aim of demonstrating the need for early intervention to remedy its consequences, and specifically children's physical fitness, since children with these difficulties tend to be sedentary and abstain from physical activity. In conclusion, we emphasize that developmental coordination disorder is not exclusively an educational problem, but is also a health problem, and that all adults involved in the child's education should be involved in its solution.
\end{abstract}

KEY WORDS: Motor competence. Developmental coordination problems. Schoolchildren. Physical activity and sport.

\section{INTRODUCCIÓN}

Hablar de escolares con problemas evolutivos de coordinación es referirnos a los niños y niñas que poseen dificultades para coordinar sus movimientos globales y/o finos en contextos muy diferentes: la escuela, sus juegos, en casa, etc. Estas dificultades les impiden desenvolverse y participar de forma competente en un conjunto amplio de actividades cotidianas, incluidas las actividades físicas y deportivas. Ya en 1980 Tom McMath ${ }^{1}$ escribía en el Physical Education Review: "Es sor- prendente la poca contribución que nuestra disciplina [educación física y ciencias del deporte] ha tenido para esclarecer este problema [la torpeza] a pesar de ser la disciplina cuya primera preocupación es el movimiento" (p. 50).

Sin duda esta misma reflexión podría aplicarse en la actualidad tanto a los médicos como a los pedagogos en nuestro contexto español: ¿quiénes se preocupan en las clases de educación física y en las canchas deportivas de esta dificultad que afecta al estilo activo de muchos escolares? 
Parece que el asunto, además de preocupar a las familias, debería reclamar la atención de los profesionales de la educación y de la salud, ya que todos en algún momento de su vida profesional han tenido alguna experiencia con estos escolares. Los médicos de cabecera y los pediatras porque han recibido la visita de padres preocupados por la forma de moverse de sus hijos, y porque el profesor de educación física sospecha de la existencia de alguna dificultad y aconseja que se le examine con más detenimiento.

Los problemas de coordinación siempre han existido y siempre han estado muy condicionados por las exigencias que existen en cada cultura. ¿Quién no recuerda a un compañero o compañera que tenía verdaderos problemas para moverse con agilidad en la clase de educación física o que trataba de inhibirse de la clase para evitar las risas del resto de los compañeros o del propio profesor? Sin duda ha llegado el momento de hablar de esta dificultad y de plantearla como un problema educativo y de salud para que, como indicaba en $1980^{1}$, "la inclusión de programas de desarrollo de la competencia motriz en la escuela sean tan válidos como los que se desarrollan para la lectura, la escritura o la aritmética” (p. 50).

\section{Moverse CON DIfICULTAD EN EL GIMNASIO}

"Isabel es una chica normal, en los estudios no va mal, saca unas notas diríamos que buenas, pero en la clase de educación física parece que se transforma, está siempre sola, cuando le propongo realizar un ejercicio siempre necesita que le anime a ello varias veces y cuando lo intenta no lo consigue realizar, lo que le provoca abandonar rápidamente. Sus compañeros me han comentado que para ella la clase de educación física es un verdadero tormento, ya que se ve incapaz de realizar las tareas que se proponen, y se cansa muy rápidamente. He hablado con su madre, le sugerí que la llevase al médico y éste la remitió a un especialista, que dictaminó que no presentaba ningún problema y que ya mejoraría con la edad" (Declaración de un profesor de educación física en Educación Secundaria Obligatoria).

Son numerosos los escolares que padecen algún tipo de discapacidad o dificultad que les hace estar menos propensos a participar en actividades físicas y deportivas de carácter exigente $^{2}$. Si consideramos que la mejora de competencia motriz y física es un elemento importante en la prevención de enfermedades, nos encontramos ante la paradoja que Ennis ${ }^{3}$ denominó de high need, low demand, es decir, es muy necesario que los escolares desde muy jóvenes practiquen actividades físicas y deportivas, pero las autoridades ofrecen pocas oportunidades en la escuela para que su práctica sea realmente efectiva ${ }^{4}$.
El hecho es que existen escolares que sobreviven en los gimnasios y que son relegados a un segundo plano, cuando no despreciados, por sus compañeros por su falta de competencia para moverse de manera coordinada en los juegos y en los deportes 5 . Son escolares que no sienten la excitación de saltar por encima de un obstáculo, de conseguir un tanto o meter una canasta, de aprender habilidades gimnásticas y mantenerse en equilibrio unos segundos sobre sus manos o de ser seleccionados los primeros para los equipos en los recreos; en definitiva, no poseen la habilidad necesaria para poderse desenvolver de forma competente con sus iguales y prefieren jugar con compañeros más pequeños o inhibirse de la práctica. Esta circunstancia les lleva a poseer una condición física menor y a una susceptibilidad a la fatiga mayor que la de sus compañeros.

Los epítetos que se han empleado para denominarlos o caracterizarlos han sido numerosos, aunque los más habituales giran alrededor de torpes, patosos o descoordinados ${ }^{6}$, y las sesiones de educación física son momentos privilegiados para constatar la gran variedad de formas de actuar con competencia o sin ella, bien sea para poder atrapar una pelota, conducir un balón con los pies o para jugar al corta-hilos. Mediante esta interacción con el medio, el escolar adquiere un conocimiento implícito y explícito sobre las acciones motrices, que le dotan de los procedimientos y recursos necesarios para una participación activa en el mundo de los juegos y los deportes, y le permiten adquirir un conjunto amplio de habilidades fundamentales para el desarrollo de un futuro estilo de vida más activo y saludable.

Tal vez sea Oseretsky ${ }^{7}$ el primer investigador que destacó la existencia de la torpeza motriz como entidad neuroconductual, lo que le llevó a su estudio y evaluación durante la década de 1920 en su laboratorio del Instituto Neurológico de Moscú, desarrollando una de las baterías más conocidas para la evaluación de lo que él denominó idiocia motriz y que ha sido empleada por médicos y psicólogos durante décadas en sus numerosas versiones y adaptaciones. En sus estudios sobre las dificultades de aprendizaje, Samuel Orton ${ }^{8}$ destacó la existencia de niños "que a menudo se retrasan en el aprendizaje incluso de movimientos simples tales como andar y correr, y tienen dificultad en aprender el empleo de sus manos y para imitar movimientos que se demuestran. Son lentos al aprender a vestirse y torpes al tratar de abotonar las prendas de vestir, atarse el cordón de sus zapatos, utilizar la cuchara, y en otras tareas sencillas" (p. 121).

Para este autor, estas dificultades expresaban un desorden neurológico de la planificación de las acciones, así como un problema de falta de reconocimiento visual de patrones espaciales. Durante varias décadas estos problemas pasaron inad- 
vertidos, hasta que en la década de 1960 se publicó en el British Medical Journal un artículo firmado por Walton, Ellis y Court ${ }^{9}$ titulado "Los niños torpes", momento en el que la profesión médica, y posteriormente psicólogos y educadores, empezaron a preocuparse por este problema. En él describían las características que presentaba un sector de la población infantil que les era remitido a sus consultas porque mostraba torpeza al moverse en su vida cotidiana y en el colegio, y no encontraban que la literatura médica se hubiera preocupado suficientemente de esta condición. En dicho artículo presentaban el estudio de 5 niños, y resaltaban que lo llamativo de sus casos era que no manifestaban alteraciones en su sistema nervioso, tales como defectos en las vías piramidal, extrapiramidal o cerebelosas, vías tan importantes para los movimientos voluntarios, y para estos autores la clave estaba en la propia organización cerebral.

No obstante, aunque estos niños mostraban un comportamiento convencional en los exámenes neurológicos habituales, frecuentemente exhibían una variedad de diferencias neurológicas cualitativamente distinguibles que fueron denominadas "signos neurológicos suaves" (soft signs). Se consideraba que la presencia de estos signos neurológicos suaves podría ser un indicador de ligeras disfunciones del cerebro, a su vez causantes de la torpeza, de ahí que se acuñara la expresión "disfunción cerebral mínima”.

Para un sector de los pediatras, estos signos suaves se convirtieron en indicadores, y los definieron como "anormalidades mínimas que aparecen en el examen neurológico al margen de otras características que puedan existir por un trastorno neurológico permanente o transitorio". Suelen considerarse asociados con las dificultades de coordinación, conducta y aprendizaje y estar referidos a la existencia de temblores, movimientos involuntarios, un tono muscular bajo, un exceso de movimiento, retrasos en el empleo de la mano dominante, sincinesias o problemas de planificación motriz (dispraxia) ${ }^{10}$; no obstante, es un asunto que sigue sometido a polémica e investigación ${ }^{11}$.

Sin duda fue Gubbay ${ }^{12}$ el que, en 1975, puso en conocimiento entre médicos y psicólogos la existencia de niños que eran mentalmente normales, que no tenían alteraciones morfológicas ni problemas diagnosticados pero que mostraban claros problemas para llevar a cabo movimientos intencionales y coordinados, separándolos de otros niños con problemas de coordinación debidos a problemas médicos diagnosticados. Para este investigador, la torpeza motriz poseía entidad suficiente para ser estudiada por sí misma, y no sólo porque formara parte de un cuadro más general de dificultad, añadiendo otro elemento de claro interés como era el papel de la cultura en su de- finición; de ahí que considerara que la torpeza motriz era una construcción social en la medida de que se es más o menos torpe en relación a unos criterios de referencia de lo que se considera un gesto coordinado ${ }^{13}$.

Sea como fuere, lo cierto es que la mayoría de los escolares que tienen dificultades para coordinar sus movimientos en el gimnasio no presentan un cuadro clínico claro que explique dichas dificultades. Como indican Larkin y Hoare ${ }^{14}$, estos niños no suelen mostrar defectos físicos en los que predomine la heterogeneidad, ya que sus comportamientos pueden ser impulsivos o pasivos; pueden ser altos o bajos, delgados o gruesos, de ahí que sea difícil encontrar unas características físicas concretas. Son niños y niñas que pueden escapar a la observación de sus padres, profesores e incluso médicos, pero que conviven con dificultades para poder desenvolverse bien en las clases de educación física y en el aprendizaje de los deportes.

Existe un consenso en denominarlos "problemas o trastornos evolutivos de coordinación motriz" (developmental coordination disorders $[\mathrm{DCD}])^{15,16}$, términos a los que adscriben las siguientes características:

- Problemas con la organización del movimiento y su estructura espaciotemporal.

- Diferencias cualitativas de sus movimientos en comparación con los realizados por sus iguales.

- Presencia de otros problemas asociados que afectan a su funcionamiento en la vida escolar o en sus actividades cotidianas.

En este artículo se utilizará de forma predominante la expresión "problemas evolutivos de coordinación motriz", con su acrónimo PECM, aunque sabemos que en medios terapéuticos se emplea también la expresión "trastornos evolutivos de la habilidad motriz" o "dispraxia evolutiva" 17 .

Un análisis de los diferentes estudios realizados sobre este sector de la población escolar manifiesta que globalmente poseen un conjunto de peculiaridades:

- Manifiestan dificultades para llevar a cabo y aprender las habilidades motrices del programa de educación física, y muestran retraso en las habilidades motrices fundamentales.

- Poseen una integridad psicofísica que les hace normales a todos los efectos, de ahí la dificultad que existe para establecer las causas de dichas dificultades.

- Su condición no es general, sino que muestra una gran heterogeneidad y especificidad. 
Si hubiera que establecer un perfil de estos escolares la tarea sería complicada, ya que, como indica Hoare ${ }^{18}$, la heterogeneidad de las dificultades es su característica más importante. Así, en su investigación encontró al menos 5 grupos de escolares con problemas de coordinación motriz: un primer grupo en el que las dificultades estaban desprovistas de problemas perceptivos; un segundo grupo en el que los problemas perceptivos y motrices estaban estrechamente relacionados; un tercer grupo que a pesar de puntuar bien en las tareas visuales, no eran competentes en las cinestésicas; un cuarto grupo cuyas dificultades se centraban en el procesamiento visual, y, por último, un grupo con un perfil mixto.

No obstante, se han establecido una serie de característi$\operatorname{cas}^{6,18,19,20}$, entre las que destacan la inconsistencia en sus actuaciones, la persistencia al llevar a cabo de forma repetitiva la misma acción motriz, la dependencia del modelo, la existencia de posibles asimetrías en las acciones de las diferentes partes corporales, los problemas de equilibrio dinámico, inestabilidad y tremor, la facilidad para caerse, la presencia de movimientos extraños y añadidos (sincinesias), la incapacidad para seguir ritmos establecidos, la incapacidad para controlar la fuerza o los problemas para planificar sus acciones. En términos de desarrollo, 6 podrían ser los indicadores más relevantes: 1) retraso en el dominio de las habilidades motrices básicas; 2) lentitud al vestirse; 3) problemas al escribir; 4) problemas con el equilibrio; 5) baja competencia en las habilidades con balón, y 6) problemas con las habilidades de locomoción.

Ante esta circunstancia, una de las primeras dificultades para superar es la relativa a su identificación y caracterización, así como el establecimiento de sus posibles causas, ya que mientras que para unos la causa se encuentra en problemas de carácter cinestésico y de su procesamiento informativo ${ }^{21} \mathrm{o}$ visual $^{12,23}$, para otros reside en la lentitud en el procesamiento y en la toma de decisiones al actuar ${ }^{24,25}$, dificultades para retener patrones visuales en lapsos cortos de tiempo ${ }^{26}$ o conocimiento sobre las acciones ${ }^{27}$.

Lo que parece un hecho cierto es que educadores, médicos y padres deben aceptar que estos escolares con PECM no han alcanzado un nivel de desarrollo motor deseable para poder desenvolverse con competencia en las clases de educación física, en sus juegos y en actividades recreativas que impliquen una actuación motriz. Son escolares que no poseen los recursos perceptivos motrices necesarios ni la condición física apropiada que les asegure el progreso en sus aprendizajes y que les permita saborear los beneficios del ejercicio físico ${ }^{28}$.

Para los padres y los profesores de educación física llama la atención el hecho de que sus habilidades motrices fundamen- tales $^{29,30}$ (andar, correr, saltar, lanzar, atrapar, etc.) se manifiesten en un estadio muy elemental para su edad. Así, por ejemplo, un reciente estudio ${ }^{31}$ ha mostrado las claras diferencias que existen al andar entre los escolares con y sin problemas evolutivos de coordinación motriz.

Están retrasados con respecto al resto de los compañeros de la clase, y esta circunstancia hace que constantemente sean observados y criticados por sus compañeros, lo que puede llevarles ante el pediatra para que evalúe su integridad o ante el psicólogo para valore su competencia intelectual. El hecho es que es extraño que el médico encuentre una causa clara, y es más habitual que el psicólogo encuentre que su cociente intelectual es normal, cuando no superior al del resto de los compañeros. No estamos ante un caso de falta de interés, de baja motivación o que no prestan la debida atención a lo que deben realizar, aunque es posible que su problema vaya acompañado de otras dificultades como las referidas a los problemas de atención, o que su interés y su motivación se vean mermados ante el fracaso continuo ${ }^{32}$.

Los factores que parecen influir en el desarrollo de esta condición de dificultad se podrían resumir en 3 grandes grupos. Por un lado, se ha indicado la existencia de algún tipo de daño cerebral al nacer, que se manifestaría en forma de signos neurológicos suaves (soft signs) como los anteriormente comenta$\operatorname{dos}^{33}$. También se han considerado las predisposiciones paternas hacia estos problemas de coordinación, o la existencia de problemas bioquímicos, y para otro sector de estudiosos lo que se destaca son los factores ambientales relacionados con lo que se ha denominado la privación ambiental ${ }^{34,35}$. En definitiva, se ha valorado la existencia de problemas neurológicos y de regiones específicas del cerebro que pudieran estar dañadas, y se han analizado los posibles problemas metabólicos, los efectos de la toxicidad de las drogas, la incompatibilidad sanguínea, la ingestión materna de drogas, las deficiencias vitamínicas y las condiciones ambientales en las que se desarrollan.

Ante estas dificultades, parece lógico pensar que el escolar pueda reaccionar de formas muy diferentes. Las posibles relaciones entre los PECM y otras dimensiones de la conducta de los escolares emanadas de diferentes estudios longitudinales ${ }^{27,36}$ han llegado a la conclusión de que los problemas evolutivos de coordinación motriz pueden ir acompañados de 5 efectos colaterales: 1) dificultades para la relación social; 2) dificultades de comportamiento; 3) baja autoestima; 4) disminución de la práctica de actividades físicas, y 6) baja condición física.

Queremos destacar este último punto -la baja condición física de estos escolares-, lo que los convierte en población de riesgo dada su tendencia a la obesidad y al sedentarismo. 


\section{SALUd Y VITALIDAD FÍSICA DE LOS ESCOLARES CON PECM}

Son escasos los estudios que han analizado la condición física de los escolares con problemas evolutivos de coordinación motriz, aunque se acepta un hecho: la baja vitalidad física que muestran los escolares con esta problemática ${ }^{37,38}$. Es fácil deducir que después de haber mostrado que los escolares con PECM tienen dificultades para coordinar sus acciones y hacer cooperar las diferentes partes de su cuerpo para poder golpear un volante con la raqueta o poder dar una voltereta en la colchoneta, sientan la tentación de no practicar, de no realizar actividades físicas, y que busquen otras actividades que les sean más gratificantes: ¿para qué sufrir con las ejercitaciones físicas si puedo jugar con el ordenador? Si éste es el pensamiento de muchos de estos escolares, lo cierto es que su repercusión en su nivel de competencia motriz y en su salud es clara, y de ahí que la mejora de la condición física sea necesaria para que tengan una infancia más activa y dinámica, y de este modo remediar lo que se ha denominado "síndrome de hipocinesia" o "de hipoactividad", y que en la actualidad preocupa a los responsables sanitarios de muchos países.

En este mismo sentido, hay autores que indican que estos niños son en general menos activos físicamente que los que no presentan dificultades de movimiento ${ }^{11,39-43}$. En un momento en que se plantea la necesidad de promocionar con más intensidad la mejora de la condición física ligada a la salud de los escolares, encontramos un sector de escolares de primaria y secundaria que no poseen los recursos motrices ni las percepciones de competencia necesarias para poder participar en los programas de condición física que pudieran desarrollarse en el colegio $^{44,45}$, y como es bien conocido, el bajo nivel de condición física se relaciona con una baja participación y se asocia con un incremento del riesgo de enfermedad cardiovascular, obesidad, desarrollo de hipertensión, osteoporosis y diabetes insulinoindependiente ${ }^{44}$. Muchos estudios han mostrado la existencia de factores de riesgo cardiovascular en niños -hipertensión ${ }^{46}$, colesterol y lipoproteínas ${ }^{47}$, obesidad ${ }^{48}$ - relacionados con el bajo nivel de práctica de actividad física ${ }^{49}$. Los niños que durante su infancia tuvieron altos factores de riesgo mantuvieron ese margen de diferencia en sentido negativo en relación a sus iguales a lo largo del tiempo. Las investigaciones más actuales ${ }^{50-54}$ han corroborado esta tendencia, ya que los escolares con problemas evolutivos de coordinación motriz no muestran el mismo patrón de actividad en el recreo que el resto de sus compañeros. Del mismo modo, otros estudios ${ }^{55}$ encontraron que durante las clases de educación física estos escolares man- tenían durante menos tiempo una frecuencia cardíaca superior a 159 pulsaciones/min (un 18,8\% del tiempo) en comparación con los que tenían un nivel de competencia motriz medio $(23,3 \%)$ o elevado $(31,4 \%$ del tiempo). Como indican Bouffard et $\mathrm{al}^{50}$, "los niños con dificultades de movimiento, en general, tienen estilos de vida que pueden poner en riesgo el desarrollo de habilidades motrices, las interacciones sociales y su estado de salud y condición física”.

Como consecuencia de esta baja condición física se ven limitados en sus aprendizajes motrices y deportivos ${ }^{56,57}$, lo que nos pone ante la tesitura de dilucidar qué influye sobre qué, ya que una baja condición física impide la práctica de las habilidades motrices necesarias para desenvolverse en las clases, y la baja competencia motriz impide que pueda participar plenamente en las actividades de condición física que pueden diseñarse y presentarse en las clases de educación física, ya que reclaman dichos patrones básicos de movimiento ${ }^{12,29,30}$. Estas dificultades para coordinar sus movimientos contribuyen a sus pobres resultados en los test motrices y de condición física, incluso aunque los realicen con el mismo esfuerzo y compromiso que el resto de los compañeros.

El reciente estudio llevado a cabo por Mata ${ }^{38}$ en la Facultad de Ciencias del Deporte de Toledo ha mostrado que la capacidad aeróbica de los escolares con PECM es inferior a la mostrada por el resto de escolares de su clase al realizar la carrera Navette. En este estudio participaron 111 escolares de 11 y 12 años a los que se les aplicó la batería Movement $\mathrm{ABC}^{58}$, de cuyos resultados se detectó la existencia de 37 escolares $(33,3 \%)$ que se encontraron por debajo del percentil 5 de las normas establecidas en la batería, lo que indica la existencia de verdaderos problemas de coordinación. Este porcentaje está en consonancia con los obtenidos en escolares de primero de secundaria $^{59}$, lo que llama la atención al estar por encima de lo que en la actualidad se considera un porcentaje habitual en las investigaciones, y que debería llevarnos a la reflexión.

Esta investigación apunta hacia la existencia de una mayor prevalencia de valores de condición aeróbica no recomendables para la salud en escolares con PECM en comparación con sus compañeros sin dificultades.

\section{A MODO DE CONCLUSIÓN Y RECOMENDACIÓN}

Probablemente la primera de las decisiones que debe adoptarse es reconocer su presencia y la tremenda cantidad de investigación que se ha llevado a cabo en la última década, y que en España es, hasta la fecha, de escaso interés. En segundo lugar, hay que aceptar su naturaleza heterogénea y la posibilidad 
de que vaya acompañada de otras dificultades, que en muchas ocasiones ocultan su verdadera naturaleza, y trabajar por la mejora de los instrumentos que favorezcan mejor su detección ${ }^{60,61}$. En tercer lugar, el impacto negativo que sobre las actitudes y participación en actividades físicas y deportivas tiene entre los escolares que las padecen. La aceptación de estas 3 premisas permitirá que todos los involucrados en la educación (docentes, padres, médicos, psicólogos, etc.) reconozcan que los PECM no desaparecen con la edad o la maduración, que persisten en la adolescencia y en la madurez y que, por lo tanto, se precisa la detección temprana y el desarrollo de programas de intervención que palien los efectos negativos que sobre el desarrollo motor de los escolares tiene, favoreciendo que el estilo de vida de estos escolares sea más activo.

No existen actividades imposibles a la hora de ser practicadas, sino programas imposibles de ser desarrollados cuando no se adaptan a las necesidades de los escolares, y en este caso la naturaleza múltiple de estas dificultades hace que actividades para favorecer la competencia motriz fina y global, el de- sarrollo de las habilidades motrices fundamentales, tan necesarias para el aprendizaje de los deportes, se conviertan en prioridades.

La labor del médico deportivo y del profesor de educación física es fundamental para que estos escolares puedan percibirse capaces de aprender y realizar las tareas que se les proponen, y de este modo desarrollar un estilo explicativo optimista de lo que les ocurre y que les haga ver que es posible mejorar en educación física y en los deportes. Estas intervenciones devolverán un sentimiento de competencia que les incitará a practicar más, lo que permitirá la mejora de cualidades físicas (fuerza, potencia, resistencia) necesarias para el desarrollo de la competencia motriz y deportiva, favoreciendo un estilo de vida más activo.

En definitiva, es necesario integrar los datos de investigación existentes en la actualidad y el conocimiento emanado de los diferentes enfoques que han acometido estas dificultades para el desarrollo de programas de intervención que ayuden a remediar esta dificultad y que hagan que deje de ser una dificultad oculta.

\section{Bibliografía}

1. McMath T. The clumsy child: A cause of concern. Phys Edu Review. 1980;3:50-63.

2. Healthy Children 2010 Report. Washington, DC: US Department of Health and Human Services, Office of Disease Prevention and Health Promotion; 2002.

3. Ennis CD. Curriculum: Forming and reshaping the vision of P.E. in a high need, low demand world of schools. Quest. 2006; 58:41-59.

4. Siedentop D, Locke L. Making a difference for P.E. What professors and practiccioners must build together. JOPERD. 1997; 68:25-33.

5. Gómez M, Ruiz LM, Mata E. Los problemas evolutivos de coordinación en la adolescencia: análisis de una dificultad oculta. Revista Internacional de Ciencias del Deporte (revista electrónica). 2006;1,3 (consultado el 10/5/2006). Disponible en: www.cafyd. com/REVISTA/index $3 . h t m l \#$.

6. Ruiz LM. Moverse con dificultad en la escuela. Introducción a los problemas evolutivos de coordinación motriz. Sevilla: Wanceulen; 2005
7. Oseretsky N. A scale for studying the motor capacity of children. J Clin Psychol. 1923;12:119-27.

8. Orton ST. Reading, writing and speech problems in children. New York: W.W. Norton; 1937.

9. Walton JN, Ellis E, Court SD. Clumsy children: developmental apraxia and agnosia. Brain. 1962;85:603-12.

10. Kurtz L. How to help a clumsy child. Strategies for young children with developmental motor concerns. London: Jessica Kingsley Publishers; 2003.

11. Fellick JM, Thompson J, Hart CA. Neurological soft signs in mainstream pupils. Arch Dis Child. 2001;85:371-4.

12. Gubbay SS. The clumsy child: a study of developmental apraxia and agnostic ataxia. London: W.B. Saunders Company; 1975.

13. Ruiz LM. Competencia motriz, problemas evolutivos de coordinación y deporte. Revista de Educación. 2004;335:21-34.

14. Larkin D, Hoare D. Out of step: Coordinating kids' movement. The University of Western Australia, Nedlands, West Australia: Active Life Foundation; 1991. 
15. American Psychiatric Association. Motor skill disorder 315.40, developmental coordination disorder. En: Diagnostic and Statistical Manual of Mental Disorders. 4th ed. Washington, DC: American Psychiatric Association; 1994. p. 53-5.

16. Organización Mundial de la Salud. Clasificación internacional de las enfermedades y problemas de salud relacionados [ICD-10]; 1992.

17. Miyahara M, Möbs I. Developmental dyspraxia and developmental coordination disorder. Neuropsychological Review. 1995; 5:245-68.

18. Hoare D. Subtypes of developmental coordination disorder. Adapted Physical Activity Quarterly. 1994;11:158-69.

19. Haubenstricker JL. Motor development in children with learning disabilities. JOPERD. 1982;5:41-3.

20. Cratty BJ. Desarrollo perceptual y motor en los niños. Buenos Aires: Paidós; 1982.

21. Laszlo JL, Bairstow PJ. Perceptual-motor behaviour. Developmental assessment and therapy. New York: Praeger; 1985.

22. Lord R, Hulme C. Perceptual judgements of normal and clumsy children. Developmental and Child Neurology. 1987; 29:815-40.

23. Ruiz LM, Mata E, Jiménez F. Percepción visual y problemas evolutivos de coordinación motriz en la edad escolar. Archivos de Medicina del Deporte. 2005;21:429-34.

24. Van Dellen T, Vaessen W, Schoemaker MM. Clumsiness: definition and selection of subjects. En: Kalverboer AF, editor. Developmental biopsychology. Ann Arbor: The University of Michigan; 1990. p. 135-52.

25. Rösblad B, Von Hofsten C. Repetitive goal-directed arm movements in children with developmental coordination disorders: role of visual information. Adapted Physical Activity Quarterly. 1994;11:190-202.

26. Dwyer T, Gibbons LE. The Australian Schools Health and Fitness Survey: Physical fitness related to blood pressure but not lipoproteins. Circulation. 1994;89:1539-44.

27. Wall AE, Reid G, Paton J. The syndrome of physical awkwardness. En: Reid G, editor. Problems in movement control. Amsterdam: Elsevier Science Publishers; 1990. p. 283-315.

28. Wright HC Children with developmental co-ordination disorder - A review. European Journal of Physical Education. 1997; 2:5-22.

29. Ruiz LM. Desarrollo motor y actividades físicas. Madrid: Gymnos; 1994.

30. Ruiz LM, Navarro F, Gutiérrez M, Linaza, JL, Graupera JL. Desarrollo, comportamiento motor y deporte. Madrid: Síntesis; 2001.

31. Deconinck FJA, De Clerck D, Savelsbergh GPJ, Van Coster R, Oostra A, Dewitte G, et al. Differences in gait between children with and without developmental coordination disorder. Motor Control. 2006;10:125-42.
32. Henderson SE, Hall D. Concomitants of clumsiness in young schoolchildren. Developmental Medicine and Child Neurology. 1982;12:178-85.

33. Cratty BJ. Clumsy child syndrome: Descriptions, evaluation and remediation. Langhorne, PA: Harwood Academic Press; 1994.

34. Morris PR, Whiting HTA. Motor impairment and compensatory education. London: G. Bell \& Sons Ltd.; 1971.

35. Sprinkle J, Hammond J. Movement deprivation and DCD children. Hobart, Australia: Proceedings of the Australian Association for Research in Education Conference; 1995.

36. Henderson, SE. Motor development and minor handicap. En: Kalverboer AF, Hopkins B, Geuze R, editors. Motor development in early and later childhood: Longitudinal approaches. Cambridge: Cambridge University Press; 1993. p. 286-306.

37. Hands B, Larkin D. Physical fitness and developmental coordination disorder. En: Cermak SA, Larkin D, editors. Developmental Coordination Disorder. Albany, NY: Delmar; 2002. p. 172-84.

38. Mata E. Condición física y percepción de competencia en escolares de $6 .^{\circ}$ curso de primaria con y sin problemas evolutivos de coordinación motriz. Trabajo DEA inédito. Facultad de Ciencias del Deporte. Universidad de Castilla-La Mancha; 2005.

39. Adler P. Momentum: A theory of social action. Beverly Hills, CA: Sage; 1981.

40. Cermak S. Developmental dyspraxia. En: Roy EA, editor. Advances in psychology. Vol. 23: Neuropsychological studies of apraxia and related disorders. New York: North Holland; 1985. p. $225-48$

41. Wall AE. Physically awkward children: A motor development perspective. En: Das JP, Mulcahy RF, Wall AE, editors. Theory and research in learning disabilities. New York: Plenum Press; 1982. p. 253-68.

42. Cantell MH, Smyth MM, Ahonen TP. Clumsiness in adolescence: Educational, motor, and social outcomes of motor delay detected at 5 years. Adapted Physical Activity Quarterly. 1994;11: 115-29.

43. Schöemaker MM, Kalverboer AF. Social and affective problems of children who are clumsy: How early do they begin? Adapted Physical Activity Quarterly. 1994;11:130-40.

44. Ward DS. Exercise for children with special needs. En: Pate RR, Hohn, RC, editors. Health and fitness through physical education. Champaing, IL: Human Kinetics; 1994. p. 99-111.

45. Poulsen AA, Ziviani JM. Can I play too? Physical activity engagement of children with developmental coordination disorders. Can J Occup Ther. 2004;71:100-7.

46. Woynarowska B, Mukherjee D, Roche F, Siervogel RM. Blood pressure changes during adolescence and subsequent blood pressure level. Hypertension. 1985;7:695-701.

47. Orchard TJ, Donahue RP, Kuller LH, Hodge PN, Drash AL. Cholesterol screening in childhood: Does it predict adult hyper- 
cholesterolemia? The Beaver County experience. J Pediatr. 1983; 103:687-91.

48. Charney E, Goodman HC, Mcbride M, Lyon B, Pratt R. Childhood antecedents of adult obesity: Do chubby infants become obese adults? N Engl J Med. 1976;296:6-9.

49. Pate RR, Baranowski T, Dowda M, Trost SG. Tracking of physical activity in young children. Med Sci Sports Exerc. 1996;28: 92-6.

50. Bouffard M, Watkinson EJ, Thompson LP, Causgrove Dunn JL, Romanow SKE. A test of the Activity Deficit Hypothesis with children with movement difficulties. Adapted Physical Activity Quarterly. 1996;13:61-73.

51. Kuiper D, Niemeijer A, Reynders K. Differences in movement versatility between children with and without movement difficulties: a pilot study. Abstract del póster presentado en DCD IV Conference, 4th Biennial Workshop on Children with DCD. Groningen, Holand. (Documento electrónico.) 1999 (consultado el 17/12/2004). Disponible en: http/www.ppsw.rug.nl/ $\sim \mathrm{dcd} /$ conference/1999/program1999.htm\#_Hlk482427508

52. Smyth MM, Anderson HI. Coping with clumsiness in the school playground: social and physical play in children with coordination impairments. British Journal of Developmental Psychology. 2000;18:398-413.

53. Thompson LP, Bouffard M, Watkinson EJ, Causgrove Dunn JL. Teaching children with movement difficulties: Highlighting the need for individualized instruction in regular education. Physical Education Review. 1994;17:152-9.

54. Cairney J, Hay J, Faught B, Mandigo J, Flouris A. Developmental coordination disorder, self-efficacy toward physical activity, and play: does gender matter? Adapted Physical Activity Quarterly. 2005;22:67-82.

55. Stratton G, Armstrong N. The relationship between physical activity levels and motor ability during physical education lessons. Journal of Sport Sciences. 1991;9:432.

56. O’Beirne C, Larkin D. Fitness characteristics of clumsy children. Póster presentado en el 8th IFAPA International Symposium. Miami, Florida: Agosto de 1991.

57. Parker HE, Larkin D. Children's co-ordination and developmental movement difficulty. En: Savelsberg G, Davids K, Vander Kamp J, Bennett SI, editors. Development of movement co-ordination in children. London: Routledge; 2003. p. 107-32.

58. Henderson SE, Sugden DA. Movement Assessment Battery for Children. UK: The Psychological Corporation, Harcourt Brace Jovanovich; 1992.

59. Gómez M. Problemas evolutivos de coordinación motriz y percepción de competencia en el alumnado de primer curso de educación secundaria obligatoria en la clase de educación física. Tesis doctoral inédita. Universidad Complutense de Madrid; 2003.

60. Ruiz LM, Graupera JL, Gutiérrez M. Observing and detecting pupils with low motor competence in school physical education: ECOMI scale in the gymnasium. International Journal of Physical Education. 2001;38:73-7.

61. Ruiz LM, Graupera JL, Gutiérrez M, Miyahara M. The assessment of motor coordination in children with the Movement ABC test: A comparative study among Japan, USA and Spain. International Journal of Applied Sport Sciences. 2003;15:22-35. 